\title{
Fator de forma artificial para povoamentos jovens de Tectona grandis em Mato Grosso
}

\author{
Ronaldo Drescher ${ }^{1}$, Allan Libanio Pelissari ${ }^{1}$, Fernando Henrique Gava ${ }^{1}$
}

${ }^{1}$ Universidade Federal de Mato Grosso (UFMT), Av. Fernando Corrêa da Costa, 2367, Boa Esperança, CEP 78060-900, Cuiabá, MT, Brasil, ronaldodrescher@gmail.com; allanpelissari@gmail.com; fhgava@hotmail.com

Resumo - Este trabalho teve como objetivo determinar o fator de forma artificial e confeccionar a tabela de fator de forma para a teca em Brasnorte e Santo Antônio do Leverger, Mato Grosso. Foram mensuradas 100 árvores e a cubagem de 162 em povoamentos de 2 a 10 anos de idade. O volume foi estimado pela fórmula de Smalian e o fator de forma pela razão entre este volume e o cilíndrico. Foram testados 16 modelos de fator de forma artificial, sendo que a equação $\ln \left(f_{1,3}\right)=0,774+0,697 \cdot \ln \left(\frac{d_{0,5}}{d^{2}}\right)-0,647 \cdot \ln \left(\frac{d_{0,1}}{d^{2}}\right)+0,199 \cdot \ln \left(\frac{1}{h \cdot d}\right)$ apresentou o melhor ajuste, com os diâmetros relativos de Hohenadl $\left(\mathrm{d}_{0,5}\right.$ e $\left.\mathrm{d}_{0,1}\right)$ estimados pelas respectivas equações: $\ln \left(d_{0,5}\right)=-1,066 \cdot \ln \left(\frac{1}{d}\right)+0,234 \cdot \ln \left(\frac{1}{h}\right) \quad$ e $\ln \left(d_{0,1}\right)=0,753-0,535 \cdot \ln \left(\frac{h}{d^{2}}\right)+0,199 \cdot \ln (h)$. A tabela de fator de forma abrangeu uma amplitude de 0,4038 a 0,6443 .

Termos para indexação: Teca, fator de forma, Smalian, Hohenadl.

\section{Form factor for young stands of Tectona grandis in State of Mato Grosso, Brazil}

\begin{abstract}
Abstratc - This study aimed to determine the artificial form factor and confection table form factor for teak in Brasnorte and Santo Antônio do Leverger, Mato Grosso State, Brazil. A hundred tress were measured and 162 were cubated in stands from 20 to 10 years of age. The volume was estimated using Smalian and form factors by the reason between the estimated and cylindrical volumes. Sixteen models of artificial the form factor werw tested, and the equation $\ln \left(f_{1,3}\right)=0,774+0,697 \cdot \ln \left(\frac{d_{0,5}}{d^{2}}\right)-0,647 \cdot \ln \left(\frac{d_{0,1}}{d^{2}}\right)+0,199 \cdot \ln \left(\frac{1}{h \cdot d}\right)$ presented the best fit with the diameter of Hohenadl ( $\mathrm{d} 0.5$ e $\mathrm{d} 0.1)$ estimated by the respective equations:

$\ln \left(d_{0,5}\right)=-1,066 \cdot \ln \left(\frac{1}{d}\right)+0,234 \cdot \ln \left(\frac{1}{h}\right)$ and $\ln \left(d_{0,1}\right)=0,753-0,535 \cdot \ln \left(\frac{h}{d^{2}}\right)+0,199 \cdot \ln (h)$. The form factor
\end{abstract} table covered a amplitude from 0.4038 to 0.6443 .

Index terms: Teak, form factor, Smalian, Hohenadl.

\section{Introdução}

A teca (Tectona grandis L.f.), espécie nativa do sudeste asiático, é uma alternativa às espécies nativas de elevado valor econômico, como o mogno (Swietenia macrophylla King) e a cerejeira (Torresea acreana Ducke), oferecendo excelentes perspectivas ao setor madeireiro, alternativa ao atendimento da reposição florestal e, consequentemente, melhores possibilidades ao suprimento sustentável da indústria de base florestal (Drescher, 2004).
Geralmente, os maiores incrementos nos povoamentos de teca são verificados em solos profundos, livres de impedimentos físicos, permeáveis, de fertilidade mediana, com $\mathrm{pH}$ maior que 5,5, baixo teor de alumínio e teor de cálcio entre 4,0 e 5,0 ppm (Takle \& Mujumdar, 1968; Yadav \& Sharma, 1968; Kaul et al., 1972; Salazar \& Albertin, 1974; Kaul et al., 1979; Hidalgo et al., 1986; Matricardi, 1989; Rondon Neto et al., 1998).

O fator de forma constitui-se numa importante prática para estimar o volume individual de árvores. $\mathrm{O}$ ato de estimar, por meio de regressão, deve ser feito em função 
do diâmetro à 1,3 m do solo e da altura total (Schneider, 1993).

O fator de forma, segundo Silva (1977) e Finger (1992), constitui-se num fator de redução do volume do cilindro para o volume real da árvore e pode ser, de acordo com Prodan et al. (1997), verdadeiro ou artificial, dependendo da altura em que for medido o diâmetro do cilindro de referência. Se o diâmetro do cilindro for medido em altura relativa, igual a $10 \%$ da altura total, o fator de forma será denominado real ou verdadeiro e, caso seja medido a uma altura absoluta, será falso ou artificial.

Prodan et al. (1997) citam que o fator de forma artificial depende de fatores genéricos, tais como a espécie, a idade do povoamento, a qualidade do sítio e a densidade do povoamento, variáveis consideradas difíceis de serem comprovadas, e também de fatores mensuráveis, como o diâmetro à $1,3 \mathrm{~m}$ do solo, a altura total, o tamanho da copa e o quociente de forma, que são medidos dentro do povoamento e estatisticamente comprovados.

Prodan et al. (1997), trabalhando com Cryptomeria japônica, demonstraram que o fator de forma tende a ficar constante, com o aumento do diâmetro e da altura, em aproximadamente 0,43 . Tendência também observada por Schneider (1984), Schneider \& Oesten (1999) e Finger (1997), com Pinus elliottii, Araucaria angustifolia, Eucalyptus grandis e Eucalyptus saligna.

No entanto, mesmo possibilitando segurança e elevada rentabilidade, como afirmam alguns investidores, o lucro dos plantios de teca só será garantido com o manejo correto da espécie, fundamentado em dados coletados e analisados dentro de padrões técnicos que possam garantir um manejo adequado.

O objetivo deste estudo foi desenvolver o fator de forma artificial, em relação às variáveis dendrométricas altura total, diâmetro à altura do peito e diâmetros relativos de Hohenadl, e a confecção de tabela de fator de forma artificial para a teca em povoamentos jovens de dois municípios do Estado de Mato Grosso.

\section{Material e métodos}

Em fevereiro de 2000, foram coletados dados de um povoamento homogêneo de teca com 50 ha, implantado em janeiro de 1994, dentro do espaçamento de $3 \mathrm{~m} \mathrm{x}$ $2 \mathrm{~m}$, na Fazenda Ribeirão da Estiva, localizada entre os paralelos $15^{\circ} 45^{\prime} \mathrm{S}$ e $16^{\circ} 00^{\prime} \mathrm{S}$ e entre os meridianos $55^{\circ} 30^{\prime} \mathrm{W}$ e $55^{\circ} 45^{\prime} \mathrm{W}$, pertencente à empresa Brasteca, no Município de Santo Antônio do Leverger, Estado de Mato Grosso, distante 98 km de Cuiabá. Em novembro de 1999 e em julho de 2000, foram coletados dados de povoamentos homogêneos da espécie com 1.850 ha, implantados em janeiro de 1990, 1992, 1993, 1994, 1995 e 1996, no espaçamento de 3 m x 2 m, na Fazenda Paraná, aproximadamente nas coordenadas $12^{\circ} 07^{\prime} \mathrm{S}$ e $58^{\circ} 00^{\prime} \mathrm{W}$, pertencente à empresa Berneck e Cia., localizada no Município de Brasnorte, região centro-norte do Estado de Mato Grosso, distante $580 \mathrm{~km}$ de Cuiabá.

O sistema de manejo, em ambas as regiões, não é definido e nem estudado. São adotadas algumas intervenções silviculturais, como por exemplo. rotação de 25 anos; desrama feita até os 4 anos e regime de desbaste com base no número de árvores por hectare $\mathrm{e}$ na área basal.

Foram mensuradas árvores em talhões não desbastados que sofreram apenas desrama no primeiro ao quarto ano, com a retirada de aproximadamente $50 \%$ da copa. Em ambas as áreas, coletou-se dados de 100 árvores alocadas em parcelas retangulares de $30 \mathrm{~m} \times 20 \mathrm{~m}\left(600 \mathrm{~m}^{2}\right)$, sendo que a idade dos troncos analisados variou de 2 a 10 anos de idade. $\mathrm{O}$ método de amostragem foi o aleatório e estratificado por idade.

Para a obtenção dos volumes observados, foram abatidas 162 árvores com medições de circunferência feitas a cada um metro ao longo do fuste, a partir de 0,3 $\mathrm{m}$. O volume foi estimado pela fórmula de Smalian e o fator de forma artificial foi obtido pela razão entre este volume e o cilíndrico.

Foram testados 16 modelos de fator de forma artificial (Tabela 1), sendo empregadas as variáveis: $\mathrm{h}$ - altura total $(\mathrm{m}) ; \mathrm{d}$ - diâmetro a $1,3 \mathrm{~m}$ do solo $(\mathrm{cm}) ; \mathrm{d}_{0, \mathrm{i}}-$ diâmetros relativos de Hohenadl $(\mathrm{cm})$ estimados por equações; e $f_{1,3}$ - fator de forma artificial. 
Tabela 1. Modelos de fator de forma ajustados.

\begin{tabular}{|c|c|}
\hline Número & Modelo \\
\hline 1 & $\ln \left(f_{1,3}\right)=b_{0}+b_{1} \cdot \ln \left(\frac{d_{0,5}}{d^{2}}\right)$ \\
\hline 2 & $\ln \left(f_{1,3}\right)=b_{0}+b_{1} \cdot \ln \left(\frac{d_{0,5}}{d^{2}}\right)+b_{2} \cdot \ln \left(\frac{d_{0,1}}{d^{2}}\right)$ \\
\hline 3 & $\ln \left(f_{1,3}\right)=b_{0}+b_{1} \cdot \ln \left(\frac{d_{0,5}}{d^{2}}\right)+b_{2} \cdot \ln \left(\frac{d_{0,1}}{d^{2}}\right)+b_{3} \cdot \ln \left(\frac{1}{h \cdot d}\right)$ \\
\hline 4 & $\ln \left(f_{1,3}\right)=b_{0}+b_{1} \cdot \ln \left(\frac{d_{0,5}}{d^{2}}\right)+b_{2} \cdot \ln \left(\frac{d_{0,1}}{d^{2}}\right)+b_{3} \cdot \ln \left(d_{0,5} \cdot d_{0,1}\right)$ \\
\hline 5 & $\ln \left(f_{1,3}\right)=b_{0}+b_{1} \cdot \ln \left(\frac{d_{0,5}}{d^{2}}\right)+b_{2} \cdot \ln \left(\frac{d_{0,7}}{d^{2}}\right)$ \\
\hline 6 & $\ln \left(f_{1,3}\right)=b_{1} \cdot \ln \left(\frac{d_{0,5}}{d^{2}}\right)+b_{2} \cdot \ln \left(\frac{d_{0,7}}{d^{2}}\right)$ \\
\hline 7 & $\mathrm{f}_{13}=\mathrm{b}_{0}+\mathrm{b}_{1} \cdot\left(\frac{\mathrm{d}_{0,5}}{\mathrm{~d}^{2}}\right)$ \\
\hline 8 & $f_{1,3}=b_{0}+b_{1} \cdot\left(\frac{d_{0,5}}{d^{2}}\right)+b_{2} \cdot\left(\frac{d_{0,3} \cdot d_{0,7}}{d^{2}}\right)$ \\
\hline 9 & $f_{1,3}=b_{1} \cdot\left(\frac{h}{d}\right)$ \\
\hline 10 & $f_{1,3}=b_{1} \cdot\left(\frac{h}{d}\right)+b_{2} \cdot\left(\frac{1}{h}\right)$ \\
\hline 11 & $f_{1,3}=b_{1} \cdot\left(\frac{n}{d}\right)+b_{2} \cdot\left(\frac{1}{h}\right)+b_{3} \cdot d_{0,7}$ \\
\hline 12 & $f_{1,3}=b_{1} \cdot\left(\frac{d_{0,1}}{d}\right)$ \\
\hline 13 & $f_{1,3}=b_{1} \cdot\left(\frac{d_{0,1}}{d}\right)+b_{2} \cdot\left(\frac{d_{0,3} \cdot d_{0,5}}{d}\right)$ \\
\hline 14 & $f_{1,3}=b_{1} \cdot\left(\frac{d_{0,1}}{d}\right)+b_{2} \cdot\left(\frac{d_{0,3} \cdot d_{0,5}}{d}\right)+b_{3} \cdot d_{0,5}$ \\
\hline 15 & $\ln \left(f_{1,3}\right)=b_{1} \cdot \ln \left(\frac{d_{0,7}}{d^{2}}\right)$ \\
\hline 16 & $\ln \left(f_{1,3}\right)=b_{1} \cdot \ln \left(\frac{d_{0,7}}{d^{2}}\right)+b_{2} \cdot \ln \left(\frac{h}{d}\right)$ \\
\hline
\end{tabular}

Os modelos foram ajustados por meio do pacote estatístico SPSS v.7.5.1 (Statistical Package for the Social Sciences, 1996). O critério para a escolha do melhor modelo obedeceu, inicialmente, o menor erro padrão da estimativa relativo $\left(\mathrm{S}_{\mathrm{yx}} \%\right)$, o maior coeficiente de determinação ajustado $\left(\mathrm{R}_{\text {ajus }}^{2}\right)$ e a distribuição gráfica dos resíduos livre de tendenciosidade. Também foi incluido o teste $\mathrm{F}$ que ajuda na tomada de decisão (Schneider, 1997). A partir do modelo selecionado, gerou-se a tabela de fator de forma artificial.

\section{Resultados e discussão}

Os resultados dos ajustes dos modelos para o fator de forma são apresentados na Tabela 2 .

Verificou-se que os modelos apresentaram baixo erro padrão de estimativa relativo $\left(\mathrm{S}_{\mathrm{yx}} \%\right)$ e coeficiente de determinação ajustado $\left(\mathrm{R}^{2}{ }_{\text {ajus }}\right)$ superior a 0,7 , exceto no modelo 1. Em geral, esses valores coincidem com os obtidos na literatura florestal para equações de fator de forma artificial de várias espécies (Higuchi, 1979; Drescher, 2004). No entanto, para a teca, não foram encontrados trabalhos relacionados ao estudo do fator de forma em sua região de origem e nem nos países onde foi implantada.

Os modelos 1, 2 e 7 foram os que apresentaram menores $\mathrm{R}_{\text {ajus }}$, respectivamente $0,659,0,720$ e 0,727 , e $\mathrm{S}_{\mathrm{yx}} \%$ de $10,61 \%, 9,63 \%$ e $9,6 \%$. No entanto, os modelos que apresentaram os maiores erros padrão de estimativa foram o 12 e o 9 , com $19,91 \%$ e $17,0 \%$, não sendo recomendados para estimar o fator de forma para a teca.

Nos modelos 9 e 10, verificou-se que as variáveis independentes diâmetro à altura do peito (d) e altura total (h), quando transformadas em inverso ou na razão $\left(\mathrm{h} \mathrm{d}^{-1}\right)$, foram melhores associadas com o fator de forma artificial, porém com altos erros padrão de estimativa, sendo $17 \%$ e $12,66 \%$, respectivamente.

Os modelos 3, 7, 8 e 11 apresentaram os melhores indicadores de precisão e ajuste em relação ao erro padrão de estimativa. A equação 3 possui quatro coeficientes e se utiliza das variáveis independentes $\mathrm{d}$ (diâmetro à 1,3 $\mathrm{m}$ do solo), $\mathrm{d}_{0,1}$ e $\mathrm{d}_{0,5}$ (diâmetros relativos de Hohenadl) e h (altura total). Essa equação apresentou $\mathrm{R}_{\text {ajus }}^{2}$ de 0,80 e $\mathrm{S}_{\mathrm{yx}}$ de $8,13 \%$, considerado um bom ajuste.

A análise dos resíduos permitiu verificar que os modelos 3 e 11 (Figura 1) mostraram um bom ajuste para o conjunto de dados e não apresentaram tendências de super ou subestimativas, com uma amplitude de dispersão dos resíduos inferior a 0,20. 
Tabela 2. Coeficientes e estatísticas dos modelos de fator de forma artificial ajustados.

\begin{tabular}{ccccccccc}
\hline $\mathbf{N}^{\mathbf{0}}$ & $\mathbf{b}_{\mathbf{0}}$ & $\mathbf{b}_{\mathbf{1}}$ & $\mathbf{b}_{\mathbf{2}}$ & $\mathbf{b}_{\mathbf{3}}$ & $\mathbf{R}_{\text {ajus }}^{\mathbf{2}}$ & $\mathbf{S}_{\mathbf{y x}}^{\mathbf{\%}}$ & $\mathbf{F}$ & $\mathbf{P r o b}>\mathbf{f}$ \\
\hline 1 & 0,167 & 0,294 & & & 0,659 & 10,61 & 303,13 & 0,000 \\
2 & 0,333 & 0,584 & $-0,283$ & & 0,720 & 9,63 & 201,18 & 0,000 \\
3 & 0,774 & 0,697 & $-0,647$ & 0,199 & 0,800 & 8,13 & 209,21 & 0,000 \\
4 & 0,501 & 0,667 & $-0,422$ & 0,056 & 0,757 & 8,96 & 163,08 & 0,000 \\
5 & 0,162 & 0,097 & 0,264 & & 0,689 & 10,21 & 170,31 & 0,000 \\
6 & & 0,100 & 0,207 & & 0,987 & 10,57 & $6.094,76$ & 0,000 \\
7 & 0,348 & 2,721 & & & 0,727 & 9,60 & 430,51 & 0,000 \\
8 & 0,369 & 2,953 & $-0,035$ & & 0,743 & 9,32 & 233,97 & 0,000 \\
9 & & 0,500 & & & 0,976 & 17,00 & $6.451,93$ & 0,000 \\
10 & & 0,275 & 2,592 & & 0,986 & 12,66 & $5.875,83$ & 0,000 \\
11 & & 0,204 & 2,689 & 0,013 & 0,991 & 10,16 & $6.110,39$ & 0,000 \\
12 & & 0,454 & & & 0,966 & 19,91 & $4.660,68$ & 0,000 \\
13 & & 0,591 & $-0,013$ & & 0,983 & 14,29 & $4.599,61$ & 0,000 \\
14 & & 0,553 & $-0,025$ & 0,024 & 0,990 & 11,12 & $5.100,75$ & 0,000 \\
15 & & 0,205 & & & 0,982 & 12,59 & $8.678,41$ & 0,000 \\
16 & & 0,205 & 0,182 & & 0,984 & 11,85 & $4.908,78$ & 0,000 \\
\hline
\end{tabular}

Resíduo Modelo 3

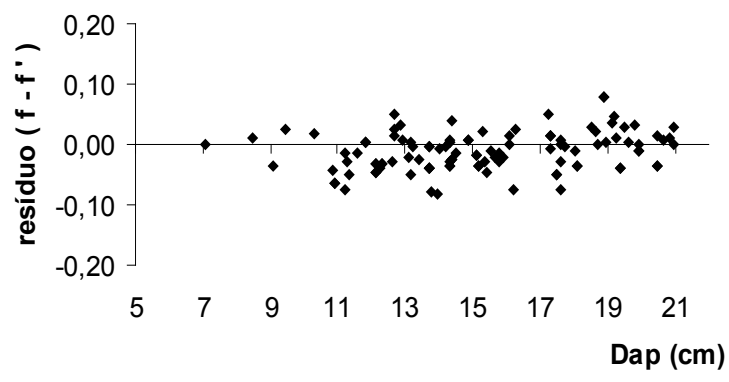

Resíduo Modelo 11

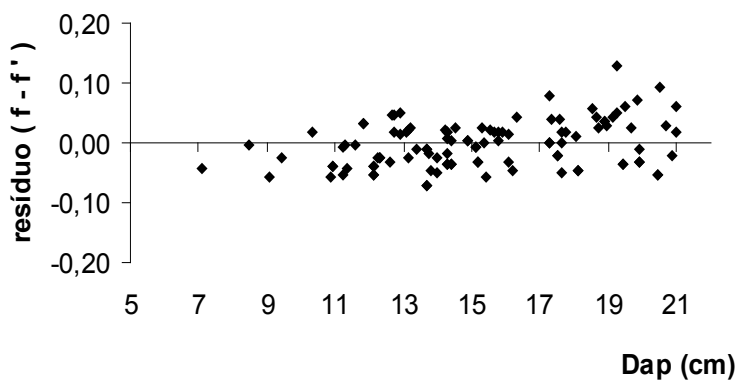

Figura 1. Distribuição dos resíduos (modelo 3 e 11) de fator de forma artificial.

Pela análise gráfica dos resíduos e dos parâmetros estatísticos, verificou-se que o modelo 3 apresentou melhor ajuste ao conjunto de dados. O modelo 8 também apresentou um bom ajuste, apenas com $\mathrm{R}_{\text {ajus }}^{2}$ inferior. Assim, o modelo selecionado foi expresso por:

$\ln \left(f_{1,3}\right)=0,774+0,697 \cdot \ln \left(\frac{d_{0,5}}{d^{2}}\right)-0,647 \cdot \ln \left(\frac{d_{0,1}}{d^{2}}\right)+0,199 \cdot \ln \left(\frac{1}{h \cdot d}\right)$
Os diâmetros relativos de Hohenadl $\left(\mathrm{d}_{0,1}\right.$ e $\left.\mathrm{d}_{0,5}\right)$ foram estimados indiretamente por meio das equações apresentadas nas Tabelas 3 e 4 .

O modelo 4 apresentou os melhores parâmetros estatísticos para o $\mathrm{d}_{0,1}$, com erro padrão de estimativa de $5,46 \%$ e coeficiente de determinação de 0,803 . Para $\mathrm{d}_{0,5}$, o modelo selecionado foi o de número 6, apresentado na Tabela 4, com $\mathrm{S}_{\mathrm{yx}}$ igual a $5,83 \%$ e $\mathrm{R}_{\text {ajus }}^{2}$ de 0,992 . 
Tabela 3. Coeficientes e estatísticas para os modelos de d0,1.

\begin{tabular}{|c|c|c|c|c|c|c|c|}
\hline Modelos & $b_{0}$ & $b_{1}$ & $b_{2}$ & $\mathbf{R}_{\text {ajus }}^{2}$ & $S_{y x} \%$ & $\mathbf{F}$ & Prob $>f$ \\
\hline $1 \quad \ln \left(d_{0,1}\right)=b_{0}+b_{1} \cdot d+b_{2} \cdot\left(\frac{h}{d}\right)$ & 2,308 & 0,058 & $-0,459$ & 0,792 & 5,61 & 298,6 & 0,00 \\
\hline $2 \ln \left(d_{0,1}\right)=b_{1} \cdot d+b_{2} \cdot\left(\frac{h}{d}\right)$ & & 0,251 & $-0,006$ & 0,985 & 9,43 & $5.140,8$ & 0,00 \\
\hline $3 \quad \ln \left(d_{0,1}\right)=b_{0}+b_{1} \cdot \ln \left(\frac{h}{d^{2}}\right)$ & 1,065 & $-0,607$ & & 0,794 & 5,59 & 602,4 & 0,00 \\
\hline $4 \quad \ln \left(d_{0,1}\right)=b_{0}+b_{1} \cdot \ln \left(\frac{h}{d^{2}}\right)+b_{2} \cdot \ln (h)$ & 0,753 & $-0,535$ & 0,199 & 0,803 & 5,46 & 319,7 & 0,00 \\
\hline $5 \quad \ln \left(d_{0,1}\right)=b_{1} \cdot \ln \left(\frac{1}{d}\right)$ & & $-1,039$ & & 0,996 & 6,08 & $4.309,7$ & 0,00 \\
\hline
\end{tabular}

Tabela 4. Coeficientes e estatísticas para os modelos de $\mathrm{d}_{0,5}$.

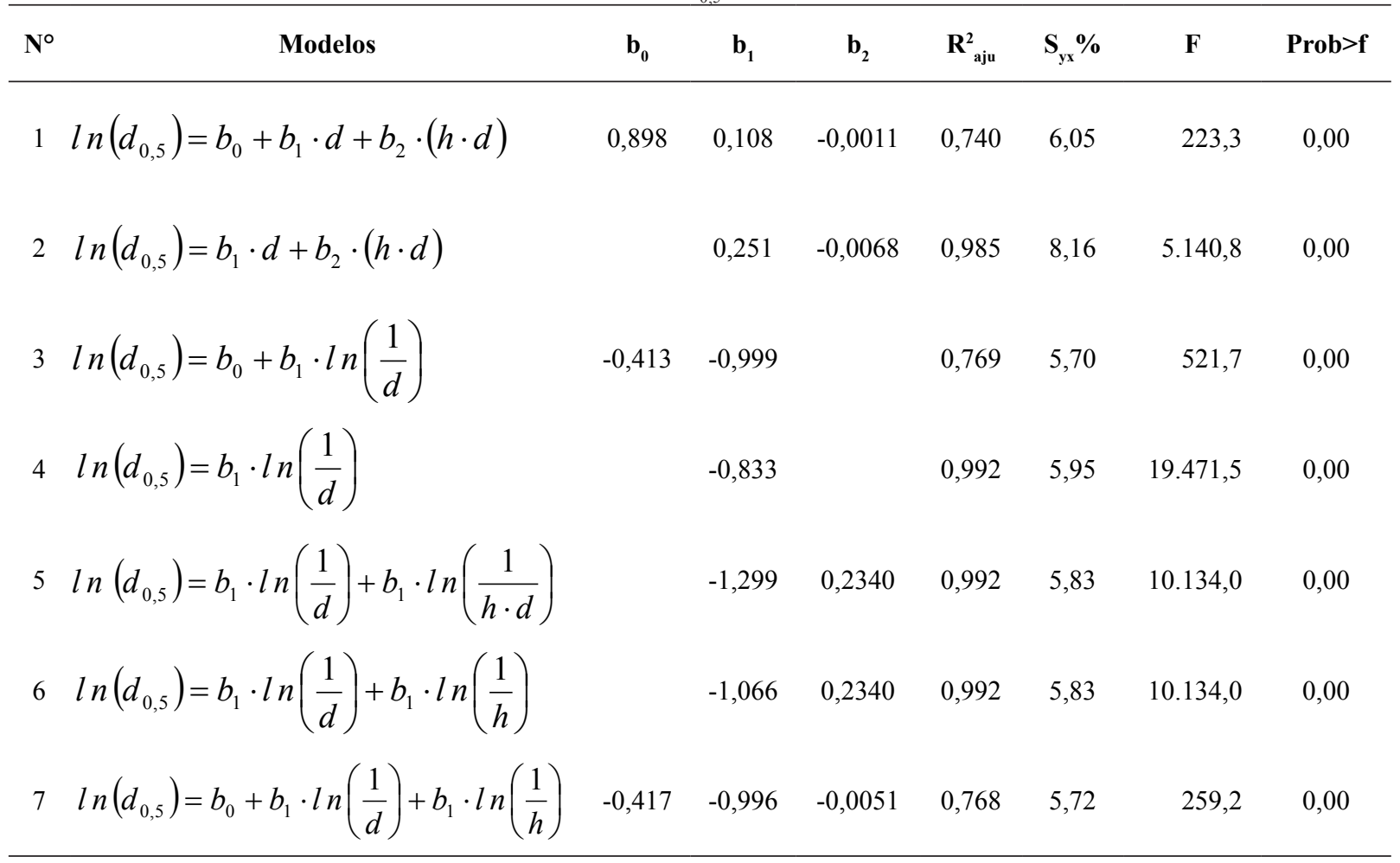

De posse do modelo de fator de forma artificial e das equações de diâmetros relativos $\mathrm{d}_{0,1}$ e $\mathrm{d}_{0,5}$, foi determinado o fator de forma artificial para a teca nos municípios de Brasnorte e Santo Antonio do Leverger, MT (Tabela 5).
Observou-se que, em árvores com diâmetro superiores a $6 \mathrm{~cm}$, os fatores de forma são menores que 0,7. Esses fatores de forma diminuem com o aumento do diâmetro até permanecerem aproximadamente constantes, tal tendência foi observada por Higuchi (1979) em povoamentos de teca na região de Cáceres, MT. 


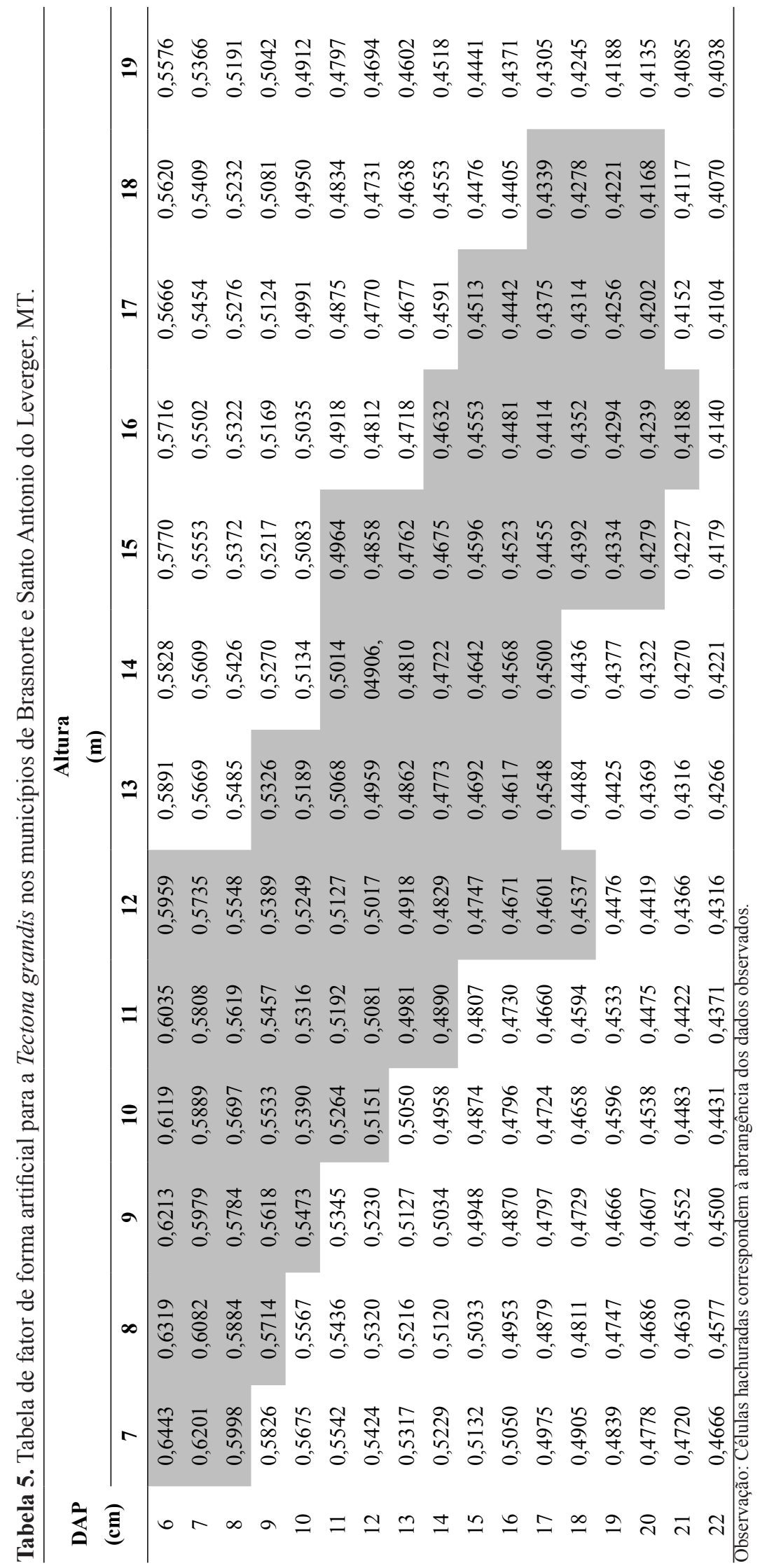




\section{Conclusões}

A equação de fator de forma artificial de melhor ajuste é a $\ln \left(f_{1,3}\right)=0,774+0,697 \cdot \ln \left(\frac{d_{0,5}}{d^{2}}\right)-0,647 \cdot \ln \left(\frac{d_{0,1}}{d^{2}}\right)+0,199 \cdot \ln \left(\frac{1}{h \cdot d}\right)$ com erro padrão de estimativa de $8,13 \%$, coeficiente de determinação de 0,80 e melhor ajuste gráfico dos resíduos, com os diâmetros relativos de Hohenadl estimados por:

$$
\begin{aligned}
& \ln \left(d_{0,5}\right)=-1,066 \cdot \ln \left(\frac{1}{d}\right)+0,234 \cdot \ln \left(\frac{1}{h}\right) \mathrm{e} \\
& \ln \left(d_{0,1}\right)=0,753-0,535 \cdot \ln \left(\frac{h}{d^{2}}\right)+0,199 \cdot \ln (h)
\end{aligned}
$$

A tabela de fator de forma artificial abrange uma amplitude de 0,4038 a 0,6443 .

\section{Referências}

DRESCHER, R. Crescimento e produção de Tectona grandis Linn F., em povoamentos jovens de duas regiões do Estado de Mato Grosso - Brasil. 2004. 133 f. Tese (Doutorado em Engenharia Florestal) - Universidade Federal de Santa Maria, Santa Maria.

FINGER, C. A. G. Fundamentos de biometria florestal. Santa Maria: UFSM/CEPEF/FATEC, 1992. 269 p.

FINGER, C. A. G. Tabelas para o manejo de Eucalyptus grandis e Eucalyptus saligna, em primeira e segunda rotação. Santa Maria: UFSM/CEPEF/FATEC, 1997. 85 p.

HIDALGO, E.; MORENO, V.; MORALES, N. Comportamiento de 15 procedências de teca (Tectona grandis) despues de cinco anos de efectuada la plantacion em Itabo, Matanzas. Revista Florestal Baracoa, Habana, v. 16, n. 1, p. 65-75, 1986.

HIGUCHI, N. Informações básicas para o manejo florestal da Tectona grandis (teca) introduzida no Alto Jaurú. Cuiabá: FUFMT, 1979. 92 p.

KAUL, O. N., GUPTA, A. C.; NEGI, J. D. S. Diagnosis of mineral deficiencies in teak (Tectona grandis L. F.) seedlings. The Indian Forester, Dehradun, v. 95, n. 3, p.173-177, 1972.
KAUL, O. N.; SHARMA, D. C.; TANDON, V. C.; SRIVASTAVA, P. B. L. Organic matter and plant nutrients in a teak (Tectona grandis L. F.) plantation. The Indian Forester, Dehradun, v. 105, n. 8 , p. $573-583,1979$.

MATRICARDI, W. A. T. Efeitos dos fatores de solo sobre o desenvolvimento da teca (Tectona grandis L. F.) cultivada na grande Cáceres - Mato Grosso. 1989. 135 f. Dissertação (Mestrado em Ciência Florestal) - Universidade de São Paulo, Piracicaba.

PRODAN, M.; PETERS, R.; COX, F.; REAL, P. Mensura Forestal. San José, 1997. 586 p.

RONDON NETO, R. M., MACEDO, R. L. G.; TSUKAMOTO FILHO, A. D. Formação de povoamentos florestais com Tectona grandis L.F. (Teca). Lavras, MG: UFLA, 1998, 33 p. (UFLA. Boletim Técnico. Série Extensão, n. 33)

SALAZAR, R.; ALBERTIN, W. Requerimentos edáficos y climáticos para Tectona grandis. Turrialba, San José, v. 24, n. 1, p. 66-71, 1974.

SCHNEIDER, P. R. Análise de regressão aplicada à Engenharia Florestal. Santa Maria: UFSM/CEPEF-FATEC, 1997. 217 p.

SCHNEIDER, P. R. Betriebswirtschaftliche und Ertragskundliche Grundlagen der Forteinrichtung in Südbrasilien am Beispiel von Pinus elliottii. Diss. d. AlbertLudwigs-Universitat Freiburg, 1984. 190 p.

SCHNEIDER, P. R. Introdução ao manejo florestal. Santa Maria: UFSM/CEPEF/FATEC, 1993. 348 p.

SCHNEIDER, P. R.; OESTEN, G. Tabelas auxiliares para o manejo florestal de Pinus elliottii Engelm. e de Araucaria angustifolia (Bert.) O. Ktze, para o planalto médio, Rio Grande do Sul. Santa Maria: UFSM/CEPEF/FATEC, 1999. 118 p.

SILVA, J. A. Biometria e estatística florestal. Santa Maria: UFSM, 1977. $235 \mathrm{p}$.

STATISTICAL PACKAGE FOR THE SOCIAL SCIENCES: Programa de computador, ambiente Windows. Chicago, 1996. Versão 7.5.1.

TAKLE, G. G.; MUJUMDAR, R. B. Increasing growth and natural regeneration of teak in Madhya Pradesh. The Indian Forest, Dehradun, v. 94, n. 12, p.897-902, 1968.

YADAV, J. S. P.; SHARMA, D. R. A Soil Investigation with reference to distribution of sal and teak in Madhya Pradesh. The Indian Forester, Dehradun, v. 94, n. 12, p. 897-902, 1968. 